setzung ist bei einem Spektrometer der verwendeten Bauart hinreichend erfüllt - :

$$
\frac{j_{\mathrm{i}}}{j_{\mathrm{k}}}=\frac{b_{\mathrm{i}}^{+}}{b_{\mathrm{k}}{ }^{+}} \frac{n_{0 \mathrm{i}}}{n_{0 \mathrm{k}}}
$$

Beim Stickstoff sind nur die Beweglichkeiten für das Molekülion, nicht aber die für das Atomion bekannt und dürften auch für das Trägergas einer StickstoffEntladung schwer zu ermitteln sein. Da jedoch die Atomkonzentration in einer Stickstoff-Entladung nur etwa $1-2 \%$ beträgt ${ }^{15}$, ist damit zu rechnen, daß das Molekülion wegen der möglichen Umladungen eine kleinere Beweglichkeit besitzt, ähnlich wie in

15 W. Groth u. P. Warneck, Z. Phys. Chem. N. F. 11, 323 [1957].
Edelgasen das Atomion ${ }^{16}$. Der Einfluß quadratischer Prozesse dagegen ist nach einer Untersuchung von SPENKE $^{17}$ von vernachlässigbarem Einfluß auf die Gültigkeit obiger Gleichung.

Unter diesen Voraussetzungen wird man die Ergebnisse der Abb. 2 als ein Maß für die Ionenkonzentrationen im Plasma ansehen können.

Diese Arbeit wurde mit Mitteln durchgeführt, die vom European $\mathrm{Office} A$ ir Research and Development Command zur Verfügung gestellt wurden.

Herrn Prof. Dr. W. Grotн bin ich für die Förderung dieser Arbeit zu großem Dank verpflichtet.

16 A. V. Phelps u. U.S. C. Brown, Phys. Rev. 86, 102 [1952].

17 E. SPenke, Z. Phys. 127, 221 [1950].

\title{
Energieübertragung bei der Fluoreszenz organischer Lösungen
}

\author{
Von Horst Knau \\ Aus dem Physikalischen Institut der Universität Gießen \\ (Z. Naturforschg. 12 a, 881-886 [1957]; eingegangen am 25. Juli 1957)
}

\begin{abstract}
Ein Phasenfluorometer zur Messung von Fluoreszenzabklingzeiten organischer Substanzen bei Elektronenanregung wird beschrieben.

Durch Messung der Abklingzeit von Anthracen und Tetracen in verschiedenen Lösungsmitteln in Abhängigkeit von der Konzentration kann auf Mechanismus und Zeitdauer des Übertragungsprozesses geschlossen werden. Es ergab sich bei großen Konzentrationen eine direkte, strahlungslose Energieübertragung durch Resonanz zwischen einem angeregten Lösungsmittelmolekül und einem Gastmolekül. Bei niedrigen Konzentrationen erfolgt zunächst eine schrittweise Wanderung von Lösungsmittelmolekül zu Lösungsmittelmolekül, bis die Energie einem Gastmolekül so nahe gekommen ist, daß sie direkt zu diesem übergehen kann.

Das Resultat wird bestätigt durch Messungen an Anthracenlösungen, deren Fluoreszenz durch Nitrobenzol gelöscht wurde. Die Diskussion wird dadurch erleichtert, daß diese Messungen sowohl bei UV-als auch bei Elektronenstoßanregung durchgeführt werden konnten.
\end{abstract}

Die stürmische Entwicklung der Kernphysik in den letzten Jahren brachte auch erhöhte Forderungen an die Leistung von Szintillationszählern mit sich. Man sah sich daher veranlaßt, Szintillatoren großer Quantenausbeute und kurzer Nachleuchtdauer zu schaffen. Einige organische Kristalle zeigen diesbezüglich recht gute Eigenschaften. Für manche Zwecke ist man aber gezwungen „feste“ oder „flüssige" Lösungen $\mathrm{zu}$ verwenden.

Wird eine solche organische Lösung mit kurzwelligem UV-Licht, $\gamma$ - oder Korpuskularstrahlung angeregt, so kann eine Übertragung der Anregungsenergie vom Lösungsmittel zum gelösten Stoff erfolgen. Dieser Vorgang ist durch Messung der Spektren und der Quantenausbeute schon vor längerer Zeit bewiesen worden. Messungen der Abklingdauer solcher
Lösungen ermöglichen es nun, auch über den $M e$ chanismus der Energieübertragung sowie über dessen Geschwindigkeit einige Aufschlüsse zu erlangen. Dies war allerdings erst durch die Entwicklung der Meßtechnik in den letzten Jahren möglich, da es sich bei diesen Lösungen um Abklingzeiten der Größenordnung $10^{-10}$ bis $10^{-8}$ sec handelt.

\section{Apparatur}

Der Anregungsteil des Fluorometers besteht aus einem auf ca. $10^{-3}$ Torr evakuierten Glasrohr, in dem eine Haarnadelkathode aus thoriertem Wolframdraht mit etwa 2 Ampere geheizt wird. Die emittierten Elektronen werden durch eine Hochspannung von 30 bis $50 \mathrm{kV}$ zur Anode beschleunigt, die auf Erdpotential liegt. Sie ist durchlöchert und mit einer $10 \mu$-Aluminium- 


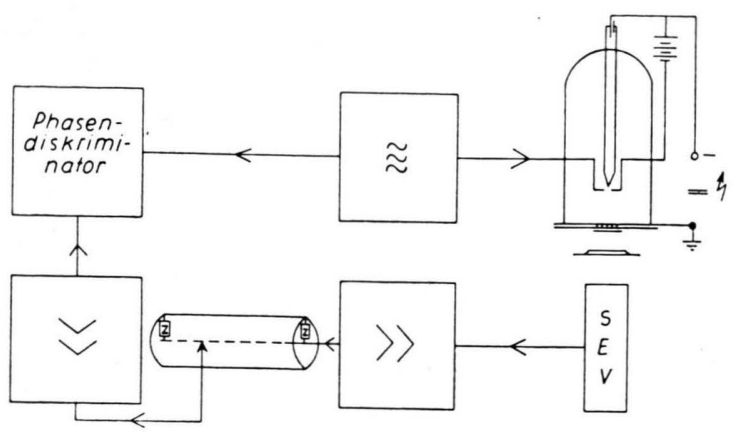

Abb. 1. Blockschaltbild.

folie abgedeckt, durch die die Elektronen in den Außenraum dringen und auf das Fluoreszenzpräparat treffen. Durch eine Hochfrequenzspannung von $10,7 \mathrm{MHz}$ am Wehnelt-Zylinder wird die Strahlintensität periodisch geändert. Das Fluoreszenzlicht oszilliert dann mit der gleichen Frequenz.

Im Meßteil des Fluorometers (siehe Blockschaltbild) wird zunächst das vom Präparat kommende optische Signal mit Hilfe eines Multipliers in ein elektrisches Signal verwandelt. Dieses durchläuft nach mehrfacher Verstärkung eine mit ihrem Wellenwiderstand abgeschlossene Verzögerungsleitung variabler Länge. Nach abermaliger Verstärkung gelangt es dann in einen Phasendiskriminator (phasenabhängiger Gleichrichter), in dem der Phasenvergleich mit dem Anregungssignal erfolgt.

Die Messung geht nun so vor sich, daß das beim Austritt der Elektronen aus dem Lenard-Fenster von der Luftfluoreszenz erzeugte Signal durch Variation der Länge des Kabels mit dem Sendersignal in Phase gebracht wird. Die von Elektronen erzeugte Fluoreszenz der Luft bei Normaldruck hat nämlich eine Abklingzeit, die innerhalb der Meßgenauigkeit der Apparatur liegt $\left(2 \cdot 10^{-10} \mathrm{sec}\right)$ und als Nullpunkt verwendet werden kann ${ }^{1}$. Danach wird das zu messende Präparat in den Strahlengang gebracht und das durch dieses erzeugte Signal ebenso mit dem Sendersignal in Phase gebracht. Aus der dazu notwendigen Änderung der Kabellänge der Verzögerungsleitung ergibt sich direkt die Phasenverschiebung zwischen Luft- und Präparatfluoreszenz. Daraus kann unter der Voraussetzung exponentiellen Abklingens die Nachleuchtdauer nach der Beziehung

$$
\tau=\frac{1}{\omega} \cdot \operatorname{tg} \varphi
$$

berechnet werden ${ }^{2}$.

$\omega=2 \pi v=$ Kreisfrequenz der Anregung; $\tau=$ Abkling. zeit.

Vergleichende Abklingzeitmessungen bei UV-Anregung wurden mit einem Fluorometer nach Schmillen durchgeführt ${ }^{3, *}$.

* Das Fluorometer zur Messung von Abklingzeiten bei UVAnregung wurde freundlicherweise von Herrn Privatdozent Dr. A. Schmillen zur Verfügung gestellt.

1 W. Hanle u. H. G. Jansen, Z. Naturforschg. 9 a, 791 [1954].

\section{Meßergebnisse}

a) Übersicht

Es wurden Lösungen von Naphthalin, Anthracen und Tetracen in verschiedenen organischen Flüssigkeiten untersucht. Die reinen Lösungsmittel selbst fluoreszieren im allgemeinen nur schwach. Aber sie zeigen, und zwar besonders die aromatischen und substituierten aromatischen Kohlenwasserstoffe (Benzol, Toluol, Xylol und Methylnaphthalin), eine gute Energieübertragung zum gelösten Stoff. Keine Energieübertragung ist zum Beispiel bei Chlor-, Brom-, Jod- und Nitrobenzol festzustellen. Die Anregungsenergie wird bei diesen Lösungsmitteln schon im Molekül selbst strahlungslos desaktiviert, wahrscheinlich in Verbindung mit einem Dissoziationsprozeß. Wie Levi und $\mathrm{F}_{\text {RANK }}{ }^{4}$ und in einer neueren Arbeit Lippert $^{5}$ gezeigt haben, übertrifft hier die Anregungsenergie die Energie der schwächsten Bindung im Molekül, so daß dieses dissoziiert.

\section{b) Zeitdauer und Mechanismus der Energie- übertragung bei großen Konzentrationen}

Durch Vergleich der Abklingzeit einer Lösung bei UV- und Elektronenstoßanregung ist es leicht möglich, die Zeitdauer des Übertragungsprozesses zu ermitteln, weil bei Elektronenstoßanregung wegen der geringen Konzentration der Fluoreszenzstoffe vorwiegend das Lösungsmittel angeregt wird, während man es bei UV-Anregung einrichten kann, daß nur

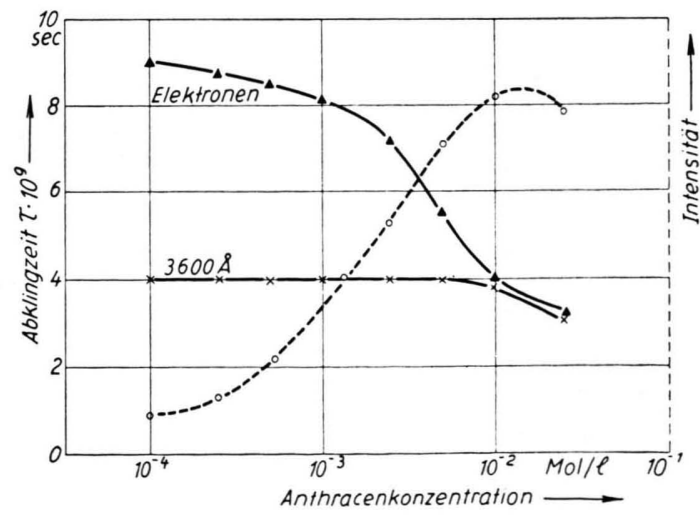

Abb. 2. Abklingzeit von Anthracen, gelöst in Benzol. Obere Kurve bei Elektronenstoßanregung, untere Kurve bei UV-Anregung. Gestrichelte Kurve: Fluoreszenzintensität bei Elektronenstoßanregung.

2 F. Duschinsky, Z. Phys. 81, 3 [1933].

3 A. Schmillen, Z. Phys. 135, 294 [1953].

4 J. Frank u. H. Levi, Z. phys. Chem. 27, 409 [1935].

5 E. Lippert, Z. phys. Chem. N. F. 2, 328 [1954]. 
die gelösten Moleküle angeregt werden. Zum Beispiel wird bei Anregung einer Lösung von Anthracen in Benzol mit UV-Licht der Wellenlänge $3100 \AA$ nur das Anthracen angeregt, da die Absorption des Benzols viel kurzwelliger liegt. Die Meßergebnisse zeigt Abb. 2. Die Abklingzeit ist unabhängig von der Konzentration der Anthracenmoleküle bis zu $10^{-2}$ $\mathrm{Mol} / l$. Bei höheren Konzentrationen macht sich die sogenannte Konzentrationslöschung bemerkbar, wie auch aus dem Verlauf der Fluoreszenzintensität bei Elektronenstoßanregung hervorgeht. Die Konzentrationslöschung kann durch kurzfristige Assoziation von einem angeregten Anthracenmolekül mit einem anderen Anthracenmolekül erklärt werden.

Wird die gleiche Lösung mit Elektronen beschossen, so ergibt sich eine völlig andere Situation. In diesem Fall wird wegen der geringen Anthracenkonzentration primär praktisch nur das Lösungsmittel Benzol angeregt. Die Anthracenmoleküle erhalten die Anregungsenergie nicht mehr direkt, sondern im wesentlichen über die Benzolmoleküle. Es ergibt sich die obere Kurve in Abb. 2, aus der folgendes entnommen werden kann: Bei UV-Anregung beträgt die Abklingzeit $4 \cdot 10^{-9} \mathrm{sec}$, und zwar unabhängig von der Konzentration. Bei Elektronenstoßanregung ergibt sich bei einer Konzentration von $10^{-4} \mathrm{Mol} / l$ eine Abklingzeit von $9 \cdot 10^{-9} \mathrm{sec}$. Die Differenz dieser beiden Zeiten, nämlich $5 \cdot 10^{-9} \mathrm{sec}$, ist die mittlere Dauer des Úbertragungsprozesses. Bei höheren Konzentrationen wird die Differenz der beiden Abklingzeiten, d. h. die Übertragungszeit, kleiner, bis sie bei $10^{-2} \mathrm{Mol} / l$ innerhalb der $\mathrm{Me}$ genauigkeit der Apparatur liegt. Das heißt in einer Zeit kleiner als $5 \cdot 10^{-10} \mathrm{sec} \mathrm{mu} \beta$ die Energie im Mittel über eine Entfernung von $30 \AA$ transportiert werden. Dieses Ergebnis steht in guter Übereinstimmung mit denen anderer Autoren ${ }^{6}$. Prozesse, die lange Zeit beanspruchen, kommen für die Erklärung nicht in Frage. Ein rascher Energieaustausch zwischen Molekülen durch Dipolwechselwirkung ist in der Literatur schon mehrfach diskutiert worden ${ }^{7}$. Mit Hilfe dieser Dipolwechselwirkung können auch die vorliegenden Meßergebnisse gut erklärt werden.

Die Energieübertragungswahrscheinlichkeit bei einem solchen Prozeß ist umgekehrt proportional der 6. Potenz des Abstandes der beiden beteiligten Moleküle. Es ist daher verständlich, daß mit sinken-

6 S. G. Cohen u. A. Weinreb, Proc. Roy. Soc., Lond. 69, 6 [1956]. - Phys. Rev. 93, 1117 [1954]. der Konzentration dieser Ubertragungsmechanismus sehr schnell unwahrscheinlich wird.

\section{Mechanismus der Energieübertragung bei niedrigen Konzentrationen}

Zunächst liegt die Vermutung nahe, daß hier eine Energieübertragung durch Absorption und Reemission vorliegt, das heißt Absorption der Benzolfluoreszenz durch die Anthracenmoleküle. Zur Klärung wurden die Spektren bei Elektronenanregung mit einem $\mathrm{Z}$ ei $ß$ schen Monochromator aufgenommen. Es ergab sich dabei, daß mit wachsender Gastkonzentration das Spektrum des Lösungsmittels immer schwächer wird, seine qualitative Form aber beibehält. Absorptionsbanden des Anthracens im Emissionsspektrum des Benzols sind nicht zu beobachten. Die Energieübertragung kann also nicht durch Absorption und Reemission erfolgen (Abb. 3).

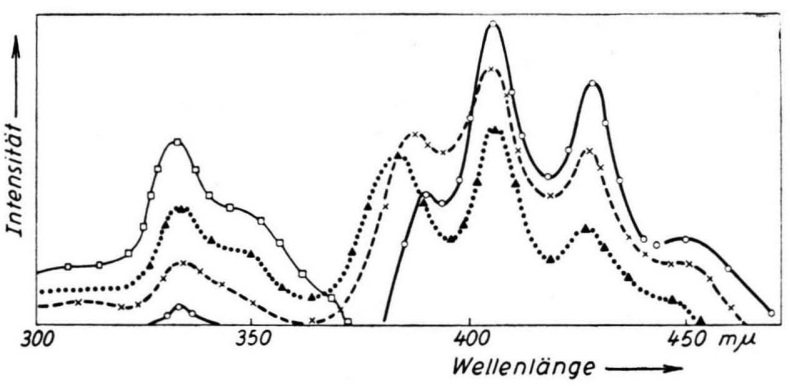

Abb. 3. Fluoreszenzspektrum von Anthracen in Benzol verschiedener Konzentration, aufgenommen bei Elektronenstoßanregung. $-\square-$ reines Lösungsmittel; $\cdots \Delta \cdots 5 \cdot 10^{-5}$ $\mathrm{Mol} / l$ Anthracen; $--\mathrm{X}--2,5 \cdot 10^{-4} \mathrm{Mol} / l$ Anthracen; $-\bigcirc-10^{-3} \mathrm{Mol} / l$ Anthracen.

Um festzustellen, in welchem Maße die Diffusion der Moleküle zur Energieübertragung beisteuert, wurde eine Konzentrationsreihe von Anthracen in einem Lösungsmittel untersucht, bei dem die Diffusionsbewegung nahezu null ist. Wird Anthracen in Polystyrol nach der Polymerisation eingelagert, so geht es keine Molekülverbindung mehr ein, sondern bleibt wie in einer Lösung unregelmäßig verteilt ${ }^{8}$. Im festen Aggregatzustand ist eine Diffusion nicht möglich. Abb. 4 zeigt, daß die Abhängigkeit der Abklingzeit von der Konzentration qualitativ die gleiche ist wie bei Anthracen - Benzol-Lösung. Dar-

\footnotetext{
7 Lit. siehe T. Förster, Fluoreszenz organischer Verbindungen, Vandenhoeck und Ruprecht, Göttingen 1951.

8 H. Hinrichs, Z. Naturforschg. 9 a, 617 [1954].
} 


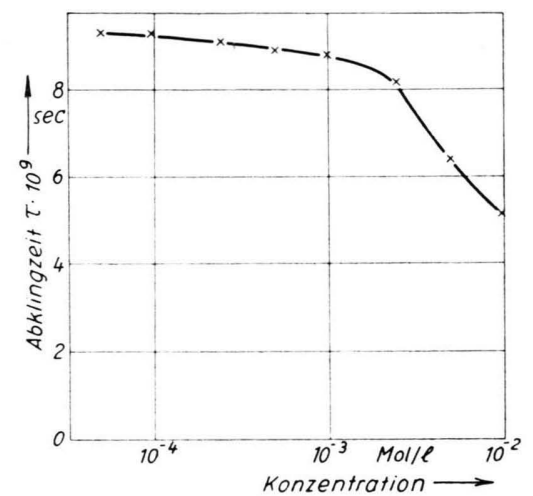

Abb. 4. Abklingzeit von Anthracen in Polystyrol bei Elektronenstoßanregung.

aus kann geschlossen werden, daß bei niedrigen Konzentrationen die Energieübertragung auch nicht der Diffusionsbewegung der Moleküle zugeschrieben werden kann.

Nachdem weder Absorption und Reemission noch Diffusion für den Energietransport verantwortlich sein kann und außerdem bei Konzentrationen $<10^{-3} \mathrm{Mol} / l$ der mittlere Abstand zwischen einem angeregten Lösungsmittelmolekül und einem Gastmolekül größer als $30 \AA$ und damit eine direkte Resonanzübertragung zwischen diesen Molekülen ebenfalls sehr unwahrscheinlich ist, wird folgende Beschreibung des Übertragungsprozesses sehr naheliegend: Die Energie muß zunächst im Lösungsmittel wandern, um in genügend große Nähe eines Gastmoleküls zu gelangen. Dies geht so vor sich, daß ein angeregtes Wirtmolekül seine Energie an ein benachbartes Wirtmolekül abgibt, dieses wieder an einen dritten Nachbarn und so fort. Auf diese Weise kann die Energie durch schrittweise Wanderung in einen so geringen Abstand zu einem Gastmolekül gelangt sein, daß sie infolge Resonanz direkt zu diesem übergehen kann. Da die Energie bei niedrigen Konzentrationen also mehrere Stationen durchlaufen muß, bevor sie vom angeregten Lösungsmittelmolekül zum nächsten Gastmolekül gelangt, wird zum Transport eine längere Zeit benötigt als bei hohen Konzentrationen, wo der Ubergang direkt erfolgen kann. Der schwache Anstieg der Abklingzeit bei Konzentrationen kleiner als $10^{-3} \mathrm{Mol} / l$ erklärt sich nun so, daß wegen des mit abnehmender Konzentration immer größer werdenden mittleren Abstandes zwischen angeregtem Molekül und Gastmolekül auch die Anzahl der von der Energie

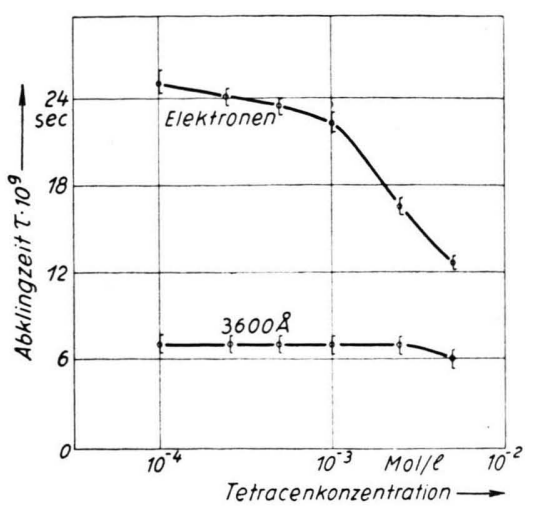

Abb. 5. Abklingzeit von Tetracen, gelöst in 1-Methylnaphthalin. Obere Kurve bei Elektronenstoßanregung, untere Kurve bei UV-Anregung.

zu durchlaufenden Zwischenstationen immer größer wird.

Qualitativ die gleichen Ergebnisse wurden auch bei anderen Lösungen erzielt. Die Absolutwerte hängen jeweils vom Lösungsmittel und dem gelösten Stoff ab. Als Beispiel soll hier nur noch Tetracen in Methylnaphthalin erwähnt werden (Abb. 5). Auch hier ist der Abfall der bei Elektronenanregung gemessenen Kurve oberhalb $10^{-3} \mathrm{Mol} / l$ deutlich zu sehen. Wegen der geringen Löslichkeit von Tetracen kann das Zusammentreffen der beiden Kurven nicht beobachtet werden. Dieser Lösung kommt deshalb besondere Bedeutung $\mathrm{zu}$, weil wegen des relativ langwelligen Absorptionsspektrums von Methylnaphthalin hier die Möglichkeit gegeben war, auch mit UV-Licht der Wellenlänge $3100 \AA$ das Lösungsmittel anzuregen. Dabei wurde der gleiche $\tau$-Wert gemessen wie bei Elektronenanregung (Tab. 1). Diese Übereinstimmung ist um so bemerkenswerter, als die Messung mit zwei verschiedenen Anordnungen erfolgte.

\begin{tabular}{|c|c|c|c|}
\hline Lösung & $\begin{array}{c}\text { Konzentration } \\
\mathrm{Mol} / l\end{array}$ & Anregung & $\begin{array}{l}\text { Abklingzeit } \\
\text { sec }\end{array}$ \\
\hline $\begin{array}{l}\text { Tetracen in } \\
\text { 1-Methyl- } \\
\text { naphthalin }\end{array}$ & $\begin{array}{l}10^{-3} \\
10^{-3} \\
10^{-3}\end{array}$ & $\begin{array}{c}\text { Elektronen } \\
\text { UV-3100 } \\
\text { UV-3600 }\end{array}$ & $\begin{array}{r}22 \cdot 10^{-9} \\
22 \cdot 10^{-9} \\
7 \cdot 10^{-9}\end{array}$ \\
\hline
\end{tabular}

Tab. 1.

\section{Die Löschwirkung von Nitrobenzol}

\section{a) Einfluß der Zähigkeit des Lösungsmittels}

Verschiedene organische Lösungsmittel sind in der Lage, die Fluoreszenz von Lösungen zu löschen, und 
zwar aus dem gleichen Grund, aus dem sie selbst fluoreszenzunfähig sind. Dies ist zum Beispiel bei der Löschung der Fluoreszenz von Anthracenlösungen durch Nitrobenzol der Fall. Die Elektronenanregungsenergie von Nitrobenzol übertrifft die Energie der schwächsten Bindung im Molekül, so daß dieses bei Anregung dissoziiert und die Energie strahlungslos abführt. Die Löschung setzt sich aus einem statischen und einem dynamischen Anteil zusammen. Ersterer wird durch solche Löschmoleküle verursacht, die mit einem Anthracenmolekül schon im Moment der Anregung „in Kontakt“ sind. Der dynamische Anteil wird durch solche Nitrobenzolmoleküle hervorgerufen, die die Anthracenmoleküle erst nach der Anregung löschen. Dieser Prozeß hat einen Abfall der Abklingzeit zur Folge.

Über die Vorgänge bei der dynamischen Löschung besteht keine einheitliche Vorstellung. Es steht zur Diskussion eine von $\mathrm{W}_{\text {AwILow }}{ }^{9}$ entwickelte Theorie, die eine Diffusion von angeregten Molekülen und Löschmolekülen zueinander vorsieht. Zur anderen Vorstellung (Förster ${ }^{7}$ ) geht man davon aus, daß ein angeregtes Anthracenmolekül auch schon über eine größere Entfernung desaktiviert werden kann, indem die Anregungsenergie durch Resonanz zum Nitrobenzol oder einem fluoreszenzunfähigen Komplex Anthracen - Nitrobenzol gelangt ${ }^{10}$.

Zur Klärung des Sachverhaltes und wegen der Möglichkeit, aus diesen Ergebnissen wieder Rückschlüsse auf die Energieübertragung in Lösungen ziehen zu können, wurde der Einfluß der Zähigkeit des Lösungsmittels sowie der Einfluß der Anregungsart auf die Löschwirkung von Nitrobenzol untersucht.

Unabhängig vom Löschmechanismus läßt sich der Verlauf einer Löschkurve durch die Formel

$$
\frac{I}{I_{0}}=\frac{1}{1+k c_{\mathrm{L}}} \quad \text { bzw. } \quad \frac{\tau}{\tau_{0}}=\frac{1}{1+k c_{\mathrm{L}}} \text { wiedergeben. }
$$

Darin bedeuten $I_{0}, \tau_{0}$ die Fluoreszenzintensität bzw. Abklingzeit der Lösung ohne Löschsubstanz, $I, \tau$ die Fluoreszenzintensität bzw. Abklingzeit mit einer Löschsubstanz, $k$ die Löschkonstante und $c_{\mathrm{L}}$ die Konzentration des Löschmittels.

Da nach der WAwilowschen Theorie die dynamische Löschung durch Diffusion der Lösch- und Fluoreszenzmoleküle im Lösungsmittel erfolgt, wäre zu

9 S. Whawlow u. W. L. Lewshin, Z. Phys. 48, 397 [1928].

10 G. Briegleb u. J. Szekalla, Z. Elektrochem. Ber. Bunsenges. Phys. Chem. 59, 184 [1955].

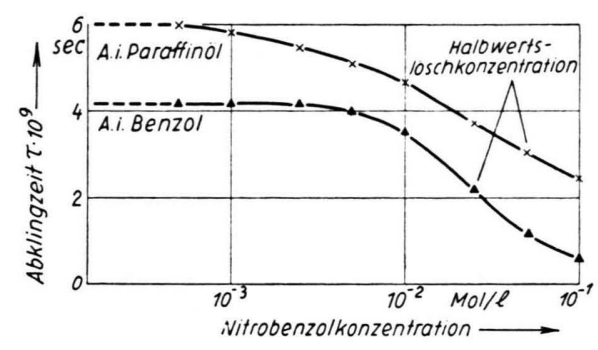

Abb. 6. Die obere Kurve gibt den Löschverlauf einer Lösung von Anthracen in Paraffinöl, die untere Kurve den von Anthracen in Benzol wieder. Die Konzentration beider Lösungen ist gleich. Angeregt wurde mit UV-Licht (3600 ̊̉).

erwarten, daß die Löschkonstante $k$ in erster Näherung umgekehrt proportional der Zähigkeit des Lösungsmittels ist. Abb. 6 gibt den Löschverlauf von Anthracen in Benzol und Anthracen in Paraffinöl in Abhängigkeit von der Löschmittelkonzentration wieder. Die Zähigkeiten von Benzol und Paraffinöl verhalten sich wie 1 : 70. Das Verhältnis der Löschkonstanten, die direkt aus den Halbwertslöschkonzentrationen entnommen werden können, ergibt sich aus den Kurven nur wie $3: 1$. Man muß deshalb annehmen, daß durch Übertragung der Anregungsenergie infolge Resonanz eines angeregten Moleküls mit einem Löschmolekül gelöscht wird. Bei einem solchen Prozeß ist wegen der großen Reichweite eine weitgehende Unabhängigkeit der Löschkonstanten von der Zähigkeit des Lösungsmittels verständlich. Ähnliche Ergebnisse erzielte auch SeElentag ${ }^{11}$ aus Messungen der Intensität der Fluoreszenz.

\section{b) Einfluß der Anregungsart auf die Löschung}

Durch Messung der Abklingzeit der Fluoreszenz von Anthracenlösungen in Abhängigkeit von der Löschmittelkonzentration kann bei UV-Anregung der Einfluß der Löschmoleküle auf die Anthracenmoleküle allein festgestellt werden (Abb. 7, untere Kurve). Bei Anregung derselben Konzentrationsreihe mit Elektronen ergibt sich eine stärkere Löschung als bei UV-Anregung. In diesem Falle wird primär das Lösungsmittel angeregt, und die Anregungsenergie kann auch schon in diesem vernichtet werden. Das Löschmolekül ist also in der Lage, die Anregungsenergie sowohl vom Lösungsmittel als auch vom Anthracen strahlungslos abzuführen. Nitrobenzol hat

11 H. Seelentag, Z. phys. Chem. N. F. 9, 373 [1956]. 


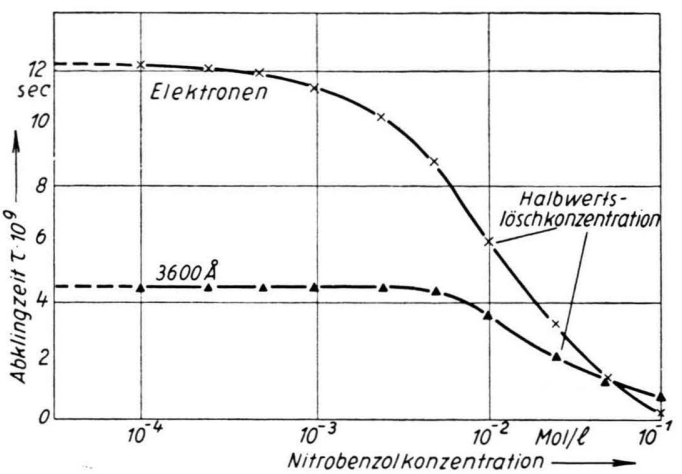

Abb. 7. Löschkurve einer Lösung Anthracen in Benzol $\left(5 \cdot 10^{-4} \mathrm{Mol} / l\right)$ bei Elektronenstoß- und UV-Anregung.

deshalb bei Elektronenstoßanregung eine stärkere Wirksamkeit als bei UV-Anregung.

Für große Anthracenkonzentrationen ergibt sich kein wesentlicher Unterschied des Löschverlaufes bei UV- und Elektronenstoßanregung (Abb. 8). Für den angeregten Zustand eines Löschmittelmoleküls steht die Möglichkeit der Energieübertragung zum Anthracenmolekül in Konkurrenz mit der Übertragung zu einem Löschmolekül. Für große Anthracenkonzentrationen überwiegt offenbar die Übertragung zu den Anthracenmolekülen. Die Nitrobenzolmoleküle müssen daher in wesentlich größerer Konzentration

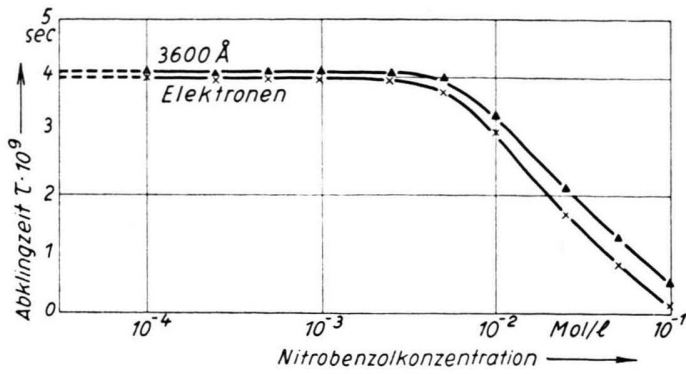

Abb. 8. Löschkurve von Anthracen in Benzol $\left(10^{-2} \mathrm{Mol} / l\right)$ bei Elektronenstoß- und UV-Anregung.

vorhanden sein, wenn in diesem Fall von ihrer Wirksamkeit auf das Lösungsmittel etwas bemerkt werden soll. In Abb. 8 ist daher erst für Nitrobenzolkonzentrationen $>10^{-2} \mathrm{Mol} / l$ eine Abweichung der beiden Kurven voneinander festzustellen.

Meinem verehrten Lehrer, Herrn Professor Dr. W. Hanle, sowie Herrn Privatdozent Dr. A. Schmillen bin ich für die fördernde Unterstützung und viele aufschlußreiche Diskussionen, zu denen auch Herr Dr. A. ScharManN wertvolle Beiträge leistete, zu großem Dank verpflichtet. Der Firma Pfe iffer (Wetzlar) und der Deutschen Forschungsgemeinschaft danke ich für die Bereitstellung von Mitteln. Die Arbeit wurde außerdem durch eine großzügige, von Herrn Professor Strahringer (Darmstadt) veranlaßte Spende unterstützt. 